E D I T O R I A L S

\title{
Is Culprit-Lesion-Only PCI in Cardiogenic Shock Still Better at 1 Year?
}

\author{
Tom Adriaenssens, M.D., Ph.D., and Frans Van de Werf, M.D., Ph.D.
}

Cardiogenic shock occurs in approximately 5\% of patients with an acute coronary syndrome usually, but not always, after ST-segment elevation myocardial infarction. If a large amount of myocardial tissue has become ischemic or injured, pump failure and reduced blood flow to vital organs occur. Urgent percutaneous coronary intervention (PCI) of the culprit lesion is currently the only therapy associated with a significant decrease in mortality. ${ }^{1}$ No other intervention with a device or pharmacologic agent has shown a significant benefit, and as a consequence, mortality has plateaued at $50 \%$ in recent years. On angiography performed during the acute phase, clinically significant lesions are often found in nonculprit vessels, a finding associated with increased mortality. ${ }^{2}$ A logical consideration, therefore, is whether treatment of these lesions during the acute phase could lower mortality. However, in the randomized Culprit Lesion Only PCI versus Multivessel PCI in Cardiogenic Shock (CULPRIT-SHOCK) trial, the rate of the primary end point of death or renal-replacement therapy at 30 days was significantly lower with culprit-lesion-only PCI than with immediate multivessel PCI. ${ }^{3}$ These results led to a downgrading of multivessel PCI to a class III B recommendation in recent European guidelines. ${ }^{4}$

In this issue of the Journal, Thiele et al. report the 1-year outcomes of the trial. ${ }^{5}$ These data are important, since it could be assumed that multivessel PCI, although harmful in the acute phase, may ultimately result in a long-term benefit. However, no significant difference in mortality or in the rate of recurrent myocardial infarction or renal-replacement therapy was found between the two approaches at 1 year. Not unexpectedly, more repeat revascularization procedures had to be performed in the culprit-lesion-only PCI group than in the multivessel PCI group (in 32.3\% of the patients vs. 9.4\%). In an exploratory landmark analysis, mortality was lower in the culpritlesion-only PCI group during the first 30 days and was similar in the two groups between 30 days and 1 year. Thus, the 1-year outcomes show that culprit-lesion-only PCI with the option of staged revascularization afterward did not increase long-term mortality.

A somewhat surprising finding of the trial is the higher rate of rehospitalization for heart failure with the culprit-lesion-only approach than with multivessel PCI (5.2\% vs. $1.2 \%)$. One explanation is that the higher rate of complete revascularization in the multivessel PCI group could have led to better left ventricular function at long-term follow-up. An alternative explanation, which was also put forward by the authors and supported by their landmark analysis, is that the culprit-lesion-only approach saved patients who would have died during the acute phase if they had received multivessel treatment, but those patients survived with poor left ventricular function and were at high risk for subsequent heart failure. A similar observation has been made in placebo-controlled trials with thrombolytic agents. ${ }^{6}$ Taken together, the results of the CULPRITSHOCK trial indicate that urgent revascularization of the culprit vessel by immediate PCI, which results in better perfusion of the jeopardized myocardium, is the key beneficial treatment to 
offer to patients who are in cardiogenic shock. The 1-year outcomes of the trial do not provide arguments to change (again) the guideline recommendations.

What can be done to further lower mortality in patients with cardiogenic shock? Shortening the time from symptom onset to PCI by streamlining prehospital care is the most important measure that can be taken, since the benefit of immediate revascularization is critically dependent on time. A large registry study showed that every 10 minutes of treatment delay resulted in 3.3 additional deaths per 100 patients who had cardiogenic shock and were treated with early PCI. ${ }^{7}$ Since substantial delays may occur before a patient gets to the catheterization laboratory, immediate administration of a thrombolytic agent should be considered in high-risk patients with acute ST-segment elevation myocardial infarction who cannot undergo immediate PCI, in order to reduce the risk of development of cardiogenic shock. The results of a recent meta-analysis that compared pharmacoinvasive treatment with standard primary PCI support this strategy. ${ }^{8}$

Are no further improvements in outcomes possible for patients who present with cardiogenic shock? Since inotropic and vasopressor agents are insufficient to interrupt the downward spiral of organ damage, hopes are set on circulatory-support devices. However, Thiele et al. have already shown that the use of an intraaortic balloon pump did not lower mortality. ${ }^{9}$ Other percutaneously inserted or surgically implanted left ventricular assist devices provide more powerful hemodynamic support, but the use of such devices has not been associated with a decrease in mortality thus far. ${ }^{10}$ Venoarterial extracorporeal membrane oxygenation is an attractive alternative option that is being used increasingly at tertiary care centers and will be tested for the first time in a large randomized trial of more than 400 patients (EURO-SHOCK). Since all these invasive interventions are expensive and their associated outcomes uncertain, we should not forget the key message of the CULPRIT-SHOCK trial: emergency revascularization in acute myocardial infarction with cardiogenic shock should be kept simple.

Disclosure forms provided by the authors are available with the full text of this editorial at NEJM.org.

From the Department of Cardiovascular Sciences, University of Leuven, Leuven, Belgium.

1. Hochman JS, Sleeper LA, Webb JG, et al. Early revascularization in acute myocardial infarction complicated by cardiogenic shock. N Engl J Med 1999;341:625-34.

2. Sanborn TA, Sleeper LA, Webb JG, et al. Correlates of oneyear survival in patients with cardiogenic shock complicating acute myocardial infarction: angiographic findings from the SHOCK trial. J Am Coll Cardiol 2003;42:1373-9.

3. Thiele H, Akin I, Sandri M, et al. PCI strategies in patients with acute myocardial infarction and cardiogenic shock. N Engl J Med 2017;377:2419-32.

4. Neumann F-J, Sousa-Uva M, Ahlsson A, et al. 2018 ESC/ EACTS guidelines on myocardial revascularization. Eur Heart J 2018 August 25 (Epub ahead of print).

5. Thiele H, Akin I, Sandri M, et al. One-year outcomes after PCI strategies in cardiogenic shock. N Engl J Med 2018;379: 1699-710.

6. Van de Werf F. Discrepancies between the effects of coronary reperfusion on survival and left ventricular function. Lancet 1989;1:1367-9.

7. Scholz KH, Maier SKG, Maier LS, et al. Impact of treatment delay on mortality in ST-segment elevation myocardial infarction (STEMI) patients presenting with and without haemodynamic instability: results from the German prospective, multicentre FITT-STEMI trial. Eur Heart J 2018;39:1065-74.

8. Vanhaverbeke M, Bogaerts K, Sinnaeve PR, Janssens L, Armstrong P, Van de Werf F. Prevention of cardiogenic shock after acute myocardial infarction. Circulation (in press).

9. Thiele H, Zeymer U, Neumann F-J, et al. Intraaortic balloon support for myocardial infarction with cardiogenic shock. N Engl J Med 2012;367:1287-96.

10. Reyentovich A, Barghash MH, Hochman JS. Management of refractory cardiogenic shock. Nat Rev Cardiol 2016;13:481-92.

DOI: 10.1056/NEJMe1810971

Copyright (c) 2018 Massachusetts Medical Society. 